\title{
Bi-Atom Electrocatalyst for Electrochemical Nitrogen Reduction Reactions
}

Cite as

Nano-Micro Lett.

(2021) 13:106

Wenchao Zhang ${ }^{1,2,3} \bowtie$, Bin-Wei Zhang ${ }^{3} \bowtie$

Received: 15 February 2021

Accepted: 28 February 2021

Published online: 7 April 2021

(C) The Author(s) 2021

\section{HIGHLIGHTS}

- A new heteronuclear bi-atom electrocatalyst has been proposed by Ma and his co-workers.

- The FeV@ $\mathrm{C}_{2} \mathrm{~N}$ bi-atom electrocatalyst achieved excellent electrochemical NRR performance.

- The FeV@ $\mathrm{C}_{2} \mathrm{~N}$ bi-atom electrocatalyst could effectively suppress the side and competing HER reaction, and thus possess better electrochemical NRR selectivity.

ABSTRACT The electrochemical nitrogen reduction reaction (NRR) to directly produce $\mathrm{NH}_{3}$ from $\mathrm{N}_{2}$ and $\mathrm{H}_{2} \mathrm{O}$ under ambient conditions has attracted significant attention due to its ecofriendliness. Nevertheless, the electrochemical NRR presents several practical challenges, including sluggish reaction and low selectivity. Here, bi-atom catalysts have been proposed to achieve excellent activity and high selectivity toward the electrochemical NRR by Ma and his co-workers. It could accelerate the kinetics of $\mathrm{N}_{2}$-to- $\mathrm{NH}_{3}$ electrochemical conversion and possess better electrochemical NRR selectivity. This work sheds light on the introduction of bi-atom catalysts to enhance the performance of the electrochemical NRR.

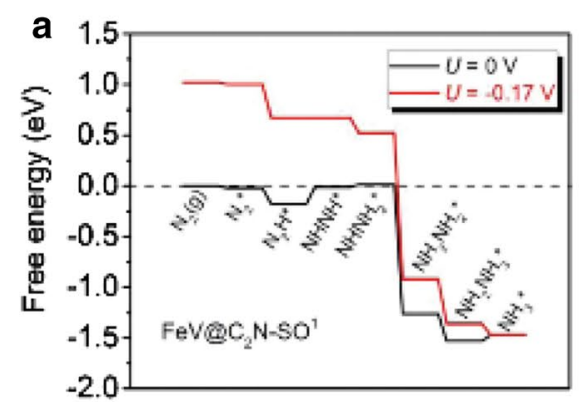

Reaction pathway

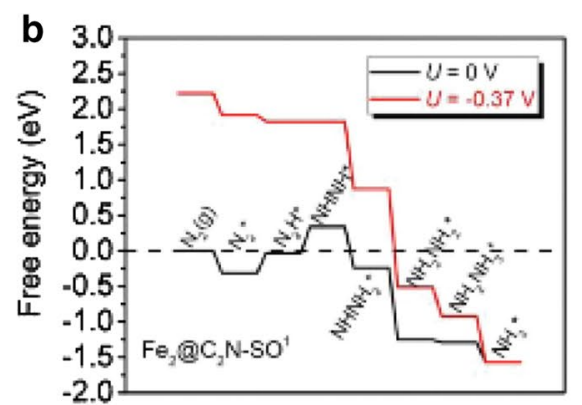

Reaction pathway

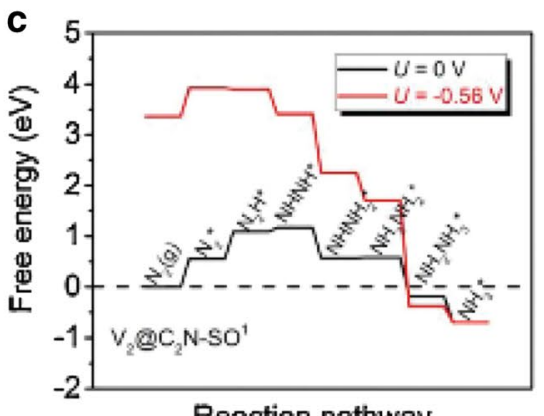

Reaction pathway

KEYWORDS Electrochemical nitrogen reduction reaction; Bi-atom catalysts; Excellent activity; High selectivity

Wenchao Zhang, wz990@uowmail.edu.au; Bin-Wei Zhang, bz370@uowmail.edu.au

1 Institute of Environmental Engineering, School of Metallurgy and Environment, Central South University, Changsha 410083, Hunan, People's Republic of China

2 Chinese National Engineering Research Center for Control and Treatment of Heavy Metal Pollution, Changsha 410083, Hunan, People's Republic of China

3 Institute for Superconducting and Electronic Materials (ISEM), School of Mechanical, Materials, Mechatronics and Biomedical Engineering, Faculty of Engineering and Information Sciences, University of Wollongong, Wollongong, NSW 2500, Australia 
The electrochemical nitrogen reduction reaction (NRR) to directly produce $\mathrm{NH}_{3}$ from $\mathrm{N}_{2}$ and $\mathrm{H}_{2} \mathrm{O}$ under ambient conditions has attracted significant attention due to its ecofriendliness compared with the traditional Haber-Bosch process [1,2]. Nevertheless, the electrochemical NRR presents several practical challenges, including sluggish reaction and low selectivity $[3,4]$. The slow kinetics is caused by the extremely strong $\mathrm{N} \equiv \mathrm{N}$ triple bond $\left(941 \mathrm{~kJ} \mathrm{~mol}^{-1}\right)$ and the great energy gap between highest occupied molecular orbital (HOMO) and the lowest unoccupied molecular orbital (LUMO) of the $\mathrm{N}_{2}$ molecule [5]. The hydrogen evolution reaction (HER) is the main side reaction responsible for the low selectivity, which shares a very close potential window with the NRR in both alkaline and acidic electrolytes $[6,7]$. Fortunately, the electrochemical NRR depends heavily on its electrocatalysts [8-10]. Therefore, advanced rational design of the electrochemical NRR electrocatalysts to achieve outstanding performance and high selectivity is urgently required [11-13]. Various NRR electrocatalysts, including metal-free catalysts, single-atom catalysts, metal nanomaterials, nitrides/oxides/sulfides/carbides, etc., have been reported with the aim of high $\mathrm{NH}_{3}$ yield since 2016 [14]. Nevertheless, a promising candidate, a heteronuclear bi-atom electrocatalyst, has been little studied for the electrochemical NRR.

Recently, Ma and co-workers [15] designed a new heteronuclear bi-atom electrocatalyst, $\mathrm{Fe}, \mathrm{V}$ co-doped $\mathrm{C}_{2} \mathrm{~N}$ $\left(\mathrm{FeV} @ \mathrm{C}_{2} \mathrm{~N}\right)$, to accelerate the kinetics of the NRR and suppress the hydrogen evolution reaction (HER), which occurs as a side reaction. This $\mathrm{FeV} @ \mathrm{C}_{2} \mathrm{~N}$ electrocatalyst achieved excellent electrochemical NRR performance. The nitrogenated holey structures in $\mathrm{C}_{2} \mathrm{~N}$ could anchor these $\mathrm{Fe}$ and $\mathrm{V}$ atoms; additionally, the unoccupied/occupied $d$ orbitals of $\mathrm{Fe}$ and $\mathrm{V}$ atoms may accept/donate electrons from/to $\mathrm{N}_{2}$ (Fig. 1a). Therefore, Fe and V atoms could be stable on the $\mathrm{C}_{2} \mathrm{~N}$ matrix and serve as active sites to electrocatalytically transform $\mathrm{N}_{2}$ into $\mathrm{NH}_{3}$. The FeV@ $\mathrm{C}_{2} \mathrm{~N}$ could weaken the $\mathrm{N} \equiv \mathrm{N}$ triple bond and increase the Bader charge difference of two chemisorbed $\mathrm{N}$ atoms, as shown in Fig. 1b-d. More importantly, the $\mathrm{FeV} @ \mathrm{C}_{2} \mathrm{~N}$ possesses the greatest ability to activate $\mathrm{N}_{2}$ compared to $\mathrm{Fe}_{2} @ \mathrm{C}_{2} \mathrm{~N}$ and $\mathrm{V}_{2} @ \mathrm{C}_{2} \mathrm{~N}$.

Furthermore, Ma and co-workers [15] proposed the mechanism of $\mathrm{N}_{2}$ reduction and free energy diagrams on side-on configurations of $\mathrm{FeV} @ \mathrm{C}_{2} \mathrm{~N}, \mathrm{Fe}_{2} @ \mathrm{C}_{2} \mathrm{~N}$, and $\mathrm{V}_{2} @ \mathrm{C}_{2} \mathrm{~N}$, and they believe that FeV @ $\mathrm{C}_{2} \mathrm{~N}$ is the most promising electrocatalyst for the NRR compared with the other two. There are only two reaction steps from $\mathrm{N}_{2} \mathrm{H}^{*}$ to $\mathrm{NHNH}^{*}$ and $\mathrm{NHNH}^{*}$ to $\mathrm{NHNH}_{2}{ }^{*}$, which are endothermic for $\mathrm{FeV} @ \mathrm{C}_{2} \mathrm{~N}$; thus, these two steps are the potential-determining step (PDS) with free energy of $0.17 \mathrm{eV}$, as shown in Fig. 2a. The $\mathrm{Fe}_{2} @$ $\mathrm{C}_{2} \mathrm{~N}$ shares the same PDS but with a higher free energy of $0.37 \mathrm{eV}$ (Fig. 2b). In $\mathrm{V}_{2} @ \mathrm{C}_{2} \mathrm{~N}$, the PDS is the formation of $\mathrm{N}_{2}{ }^{*}$, with the greatest free energy of $0.56 \mathrm{eV}$ (Fig. 2c). The HER, as a competing and side reaction, was also investigated for these three samples. The calculated results indicated that $\mathrm{FeV} @ \mathrm{C}_{2} \mathrm{~N}$ and $\mathrm{Fe}_{2} @ \mathrm{C}_{2} \mathrm{~N}$ have better NRR selectivity. $\mathrm{V}_{2} @$ $\mathrm{C}_{2} \mathrm{~N}$, because of the competing HER, is not a good candidate for the electrochemical NRR.

In sum, $\mathrm{Ma}$ and co-workers proposed $\mathrm{FeV} @ \mathrm{C}_{2} \mathrm{~N}$ as an outstanding heteronuclear bi-atom electrocatalyst for the electrochemical NRR, with high activity and better selectivity. It could enhance the kinetics of $\mathrm{N}_{2}$-to- $\mathrm{NH}_{3}$ electrochemical conversion with a low potential PDS of $-0.17 \mathrm{~V}$. Moreover, this $\mathrm{FeV} @ \mathrm{C}_{2} \mathrm{~N}$ electrocatalyst could effectivity suppress the side and competing HER reaction, and thus possess better electrochemical NRR selectivity. This work sheds light on the introduction of heteronuclear bi-atom electrocatalysts to enhance the performance of the electrochemical NRR and opens a new way to understand the electrochemical NRR mechanism.

In the future, two possible prospects could be effective approaches to optimize the electrocatalysts with the aim of improved NRR activity and selectivity, and reveal the mechanisms of the electrochemical NRR as well. Firstly, theoretical calculations could be employed to predict potential NRR electrocatalysts and provide various types of optimization guidance to the experiments. For example, high-throughput computing can identify the poisoning and decomposition of electrocatalysts under electrochemical conditions, including the $\mathrm{pH}$ and the electrolyte effect, which can provide a deeper insight into the mechanism under real operation conditions. On the other hand, advanced characterization, including in-situ and operando atomic-resolution transmission electron microscopy and X-ray absorption spectroscopy, can be developed to identify the real active sites and 
(a)

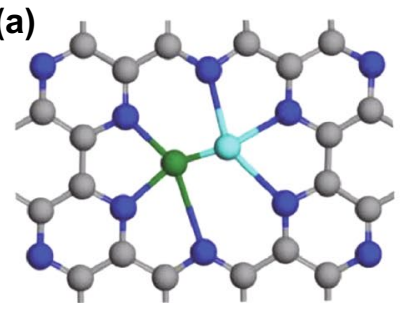

(b)

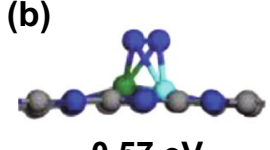

$-0.57 \mathrm{eV}$

(1)

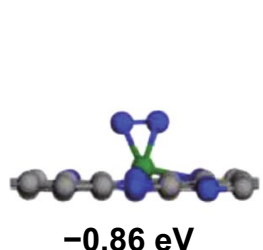

(1)

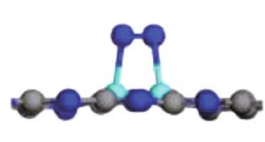

$0.01 \mathrm{eV}$

(1)

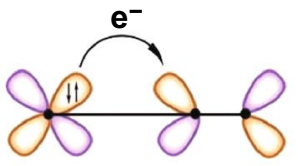

$\mathrm{M} \rightarrow \mathrm{N} \equiv \mathrm{N}$

occupied empty

d orbital $\pi^{*}$ orbital

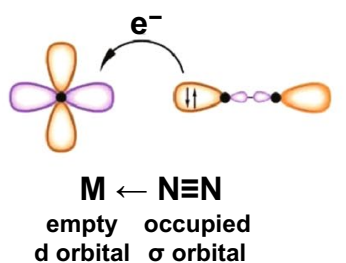

d orbital $\sigma$ orbital $\downarrow \uparrow=$ single electrons of opposite spin

$Q=$ orbital lobes of opposite phase

OC ON OFe OV

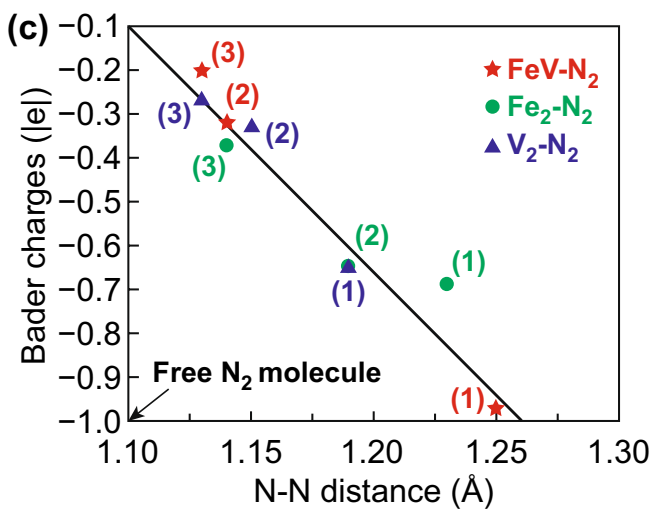

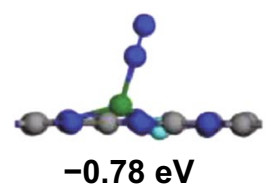

(2)

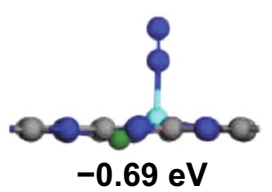

(3)

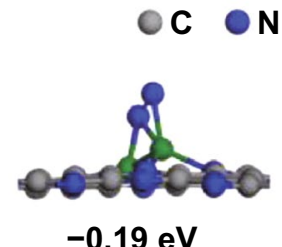

(2)

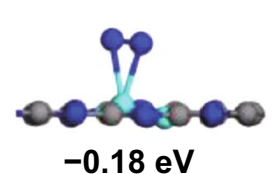

(2)

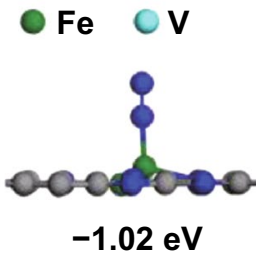

(3)

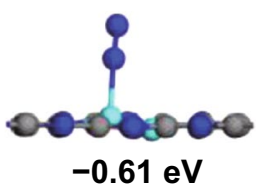

(3)

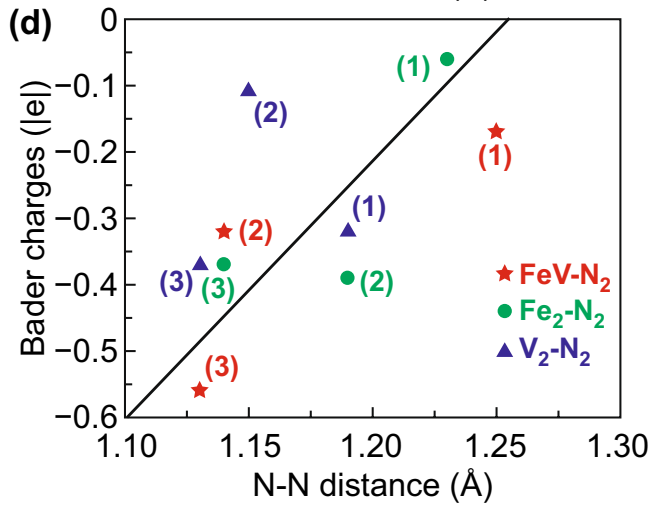

Fig. 1 a Optimized structures of $\mathrm{FeV}$ anchored on $\mathrm{C}_{2} \mathrm{~N}$ substrate and simplified schematic diagram of the bonding between the transition metal and $\mathrm{N}_{2}$. b Optimized structures and corresponding adsorption energies of $\mathrm{N}_{2}$ adsorption on $\mathrm{FeV} @ \mathrm{C}_{2} \mathrm{~N}, \mathrm{Fe}_{2} @ \mathrm{C}_{2} \mathrm{~N}$, and $\mathrm{V}_{2} @ \mathrm{C}_{2} \mathrm{~N}$. $\mathbf{c}$ Relationship between Bader charges of adsorbed $\mathrm{N}_{2}$ and $\mathrm{N}-\mathrm{N}$ bond lengths. $\mathbf{d}$ Relationship between Bader charge difference of two adsorbed $\mathrm{N}$ atoms and $\mathrm{N}-\mathrm{N}$ bond lengths [15]. Copyright 2020 Elsevier

composite evolution of the electrical double layer. With the significant efforts that have been made in the past few years, the electrochemical NRR appears promising to replace the traditional Haber-Bosch process to produce $\mathrm{NH}_{3}$. Nevertheless, a reproducible and excellent electrochemical NRR catalyst is still expected to be proposed as a standard electrocatalyst, due to the doubt that has arisen on the actual
NRR performance. A benchmarking protocol to accurately quantify the electrochemical NRR activity and selectivity should be established. We believe that, with much effort, the fundamental issues and technological drawbacks will be addressed in the not-too-distant future, and the electrochemical NRR can play an important role in $\mathrm{NH}_{3}$ yield. 

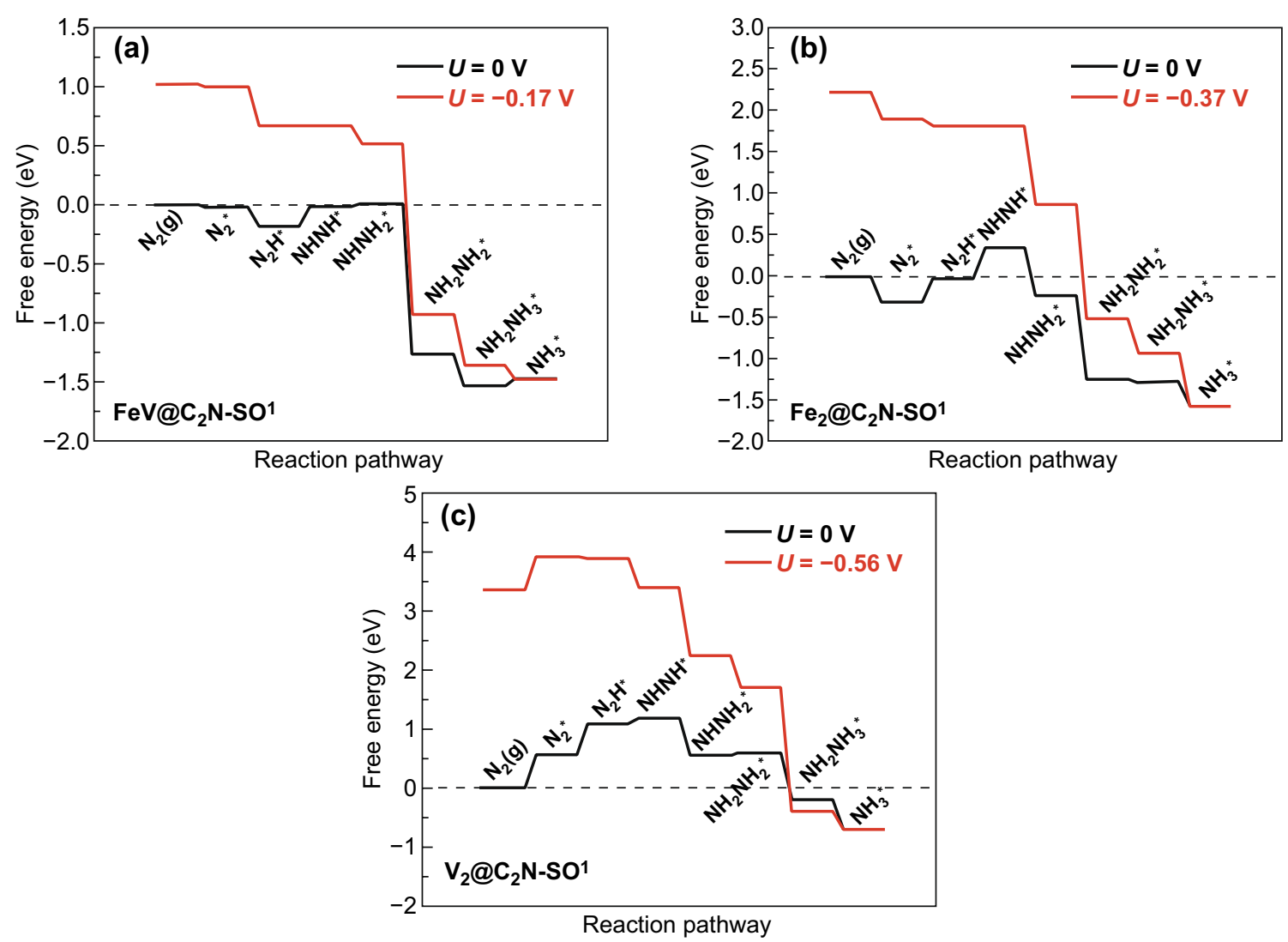

Fig. 2 a-c Free energy diagrams of the three electrocatalysts [15]. Copyright 2020 Elsevier

Open Access This article is licensed under a Creative Commons Attribution 4.0 International License, which permits use, sharing, adaptation, distribution and reproduction in any medium or format, as long as you give appropriate credit to the original author(s) and the source, provide a link to the Creative Commons licence, and indicate if changes were made. The images or other third party material in this article are included in the article's Creative Commons licence, unless indicated otherwise in a credit line to the material. If material is not included in the article's Creative Commons licence and your intended use is not permitted by statutory regulation or exceeds the permitted use, you will need to obtain permission directly from the copyright holder. To view a copy of this licence, visit http://creativecommons.org/licenses/by/4.0/.

\section{References}

1. Y. Fu, P. Richardson, K. Li, H. Yu, B. Yu et al., Transition metal aluminum boride as a new candidate for ambient-condition electrochemical ammonia synthesis. Nano Micro Lett. 12, 65 (2020). https://doi.org/10.1007/s40820-020-0400-z

2. J.C. Tang, S.-Z. Qiao, How to explore ambient electrocatalytic nitrogen reduction reliably and insightfully. Chem. Soc. Rev. 48, 3166-3180 (2019). https://doi.org/10.1039/C9CS00280D
3. B.-W. Zhang, Y.-X. Wang, S.-L. Chou, H.-K. Liu, S.-X. Dou, Fabrication of superior single-atom catalysts toward diverse electrochemical reactions. Small Methods 3, 1800497 (2019). https://doi.org/10.1002/smtd.201800497

4. J.G. Chen, R.M. Crooks, L.C. Seefeldt, K.L. Bren, R.M. Bullock et al. Beyond fossil fuel-driven nitrogen transformations. Science 360, 6611 (2018). https://doi.org/10.1126/science. aar6611

5. S.L. Foster, S.I.P. Bakovic, R.D. Duda, S. Maheshwari, R.D. Milton et al., Catalysts for nitrogen reduction to ammonia. Nat. Catal. 1, 490-500 (2018). https://doi.org/10.1038/ s41929-018-0092-7

6. B.-W. Zhang, H.-L. Yang, Y.-X. Wang, S.-X. Dou, H.-K. Liu, A comprehensive review on controlling surface composition of Pt-based bimetallic electrocatalysts. Adv. Energy Mater. 8, 1703597 (2018). https://doi.org/10.1002/aenm.201703597

7. B.-W. Zhang, T. Sheng, Y.-X. Wang, X.-M. Qu, J.-M. Zhang et al., Platinum-cobalt bimetallic nanoparticles with Pt skin for electro-oxidation of ethanol. ACS Catal. 7, 892-895 (2017). https://doi.org/10.1021/acscatal.6b03021

8. B.-W. Zhang, L. Ren, Y.-X. Wang, Y. Du, L. Jiang et al., New monatomic layer clusters for advanced catalysis materials. Sci. China Mater. 62, 149-153 (2019). https://doi.org/10.1007/ s40843-018-9317-7 
9. B.-W. Zhang, T. Sheng, Y.-D. Liu, Y.-X. Wang, L. Zhang et al., Atomic cobalt as an efficient electrocatalyst in sulfur cathodes for superior room-temperature sodium-sulfur batteries. Nat. Commun. 9, 4082 (2018). https://doi.org/10.1038/ s41467-018-06144-x

10. B.H.R. Suryanto, H.-L. Du, D. Wang, J. Chen, A.N. Simonov et al., Challenges and prospects in the catalysis of electroreduction of nitrogen to ammonia. Nat. Catal. 2, 290-296 (2019). https://doi.org/10.1038/s41929-019-0252-4

11. B.-W. Zhang, T. Sheng, Y.-X. Wang, S. Chou, K. Davey et al., Long-life room-temperature sodium-sulfur batteries by virtue of transition-metal-nanocluster-sulfur interactions. Angew. Chem. Int. Ed. 131, 1498-1502 (2019). https://doi.org/10. 1002/anie.201811080

12. H.-P. Jia, E.A. Quadrelli, Mechanistic aspects of dinitrogen cleavage and hydrogenation to produce ammonia in catalysis and organometallic chemistry: relevance of metal hydride bonds and dihydrogen. Chem. Soc. Rev. 43, 547-564 (2014). https://doi.org/10.1039/C3CS60206K

13. X. Tang, Z. Wei, Q. Liu, J. Ma, Strain engineering the D-band center for Janus MoSSe edge: Nitrogen fixation. J. Energy Chem. 33, 155-159 (2019). https://doi.org/10.1016/j.jechem. 2018.09.008

14. G. Qing, R. Ghazfar, S.T. Jackowski, F. Habibzadeh, M.M. Ashtiani et al., Recent advances and challenges of electrocatalytic $\mathrm{n}_{2}$ reduction to ammonia. Chem. Rev. 120, 5437-5516 (2020). https://doi.org/10.1021/acs.chemrev.9b00659

15. Z. Wei, J. He, Y. Yang, Z. Xia, Y. Feng et al., Fe, V-co-doped $\mathrm{C}_{2} \mathrm{~N}$ for electrocatalytic $\mathrm{N}_{2}$-to- $\mathrm{NH}_{3}$ conversion. J. Energy Chem. 53, 303-308 (2021). https://doi.org/10.1016/j.jechem. 2020.04.014 